\title{
An implementation of modified nodal array approach in designing a readout circuit for piezoresistive pressure sensor array
}

\begin{abstract}
In this paper, we modified the Nodal Array Approach (NAA) in designing readout circuit for piezoresistive pressure sensor array to include more sensors in a network and rearranging the topology of the variable resistors for more efficiency and accuracy in producing the results. In order to maximize the number of sensors that can be read in a single network, NAA's reading technique also needs to be modified and extended to more than 4 iterations readings. Furthermore, the modified topology of variable resistors locations has indirectly remove its previous limitation of uniqueness and permutation. An additional work has been done in dividing the sensor's resistance value into range group in order to suit the pressure sensor's behavior characteristic. For any sensor that operating in a specific range of operation, the results can achieved lower than 5\% of calculation error by comparing to its real value.
\end{abstract}

Keyword: Resistive readout circuit; Resistive sensor array; Foot plantar application 